\title{
On the Origin of Light-Induced Changes in the Proton Magnetic Relaxation Rate of Chloroplast Thylakoid Membrane Suspensions ${ }^{1}$
}

\author{
H. H. ROBINSON, $\dagger$ R. R. SHARP,* AND C. F. YOCUM ${ }^{* *}$ \\ *Department of Chemistry and + Division of Biological Sciences, University of Michigan, \\ Ann Arbor, Michigan 48109
}

Received June 2, 1980

\begin{abstract}
The spin-lattice relaxation rate of solvent protons in suspensions of chloroplast thylakoid membranes undergoes a large transient depression following illumination in white light. This change appears to require the presence of chelatable paramagnetic ions; it is absent in chloroplasts exposed to $1 \mathrm{mM}$ EDTA during the homogenization step of the isolation procedure, but reappears when $50 \mu \mathrm{M} \mathrm{MnCl}_{2}$ is added to these suspensions. Conditions that inhibit light-induced $R_{1}$ changes are (i) anaerobiosis, (ii) inhibition of plastocyanin function by $\mathrm{Hg}^{+2} / \mathrm{CN}$, and (iii) the presence of superoxide dismutase. These observations suggest that chemical oxidation of nonfunctional Mn(II) by superoxide ion, which is generated under aerobic conditions by autooxidizable acceptors of Photosystem $I$, is responsible for the phenomenon. This interpretation was confirmed by experiments involving superoxide generation in the dark, using the NADPH-driven diaphorase activity of ferredoxin-NADPreductase with benzylviologen as an autooxidizable acceptor.
\end{abstract}

Nuclear magnetic relaxation times, $T_{1}$ and $T_{2}$, of solvent protons have been used extensively as probes of paramagnetic metal ions bound to soluble enzymes (1-3) and offer similar potential as probes of membrane-bound redox proteins (4-7). When bound in sites of reorientationally restricted mobility $(1,5), \mathrm{Mn}$ (II) provides an extremely efficient relaxation pathway for solvent protons, provided that the coordination sphere of the ion is accessible to the solvent. In chloroplast suspensions, addition of $\mathrm{MnCl}_{2}$ to a final concentration of $25 \mu \mathrm{M}$ produces a doubling of the control relaxation rate due to binding of the ion to the thylakoid membrane $(5,6)$. This concentration is comparable to the manganese content of the oxygen-evolving complex in concentrated $(2-3 \mathrm{mg} \mathrm{chl} / \mathrm{ml})^{2}$ thylakoid

\footnotetext{
1 This research was supported by a grant from the National Science Foundation (PCM78-7909) to C.F.Y.

${ }^{2}$ Abbreviations used: Chl, chlorophyll; DCMU, 3-(3,4-dichlorophenyl)-1,1'-dimethylurea; MV, methylviologen; $\mathrm{BV}$, benzylviologen; $\mathrm{BSA}$, bovine serum albumin; Tris, tris(hydroxymethyl)aminomethane; PSI, PsII, Photosystem I, II.
}

suspensions, and it has been proposed that a variety of relaxivity changes previously reported in chloroplast suspensions monitor the oxidation states of manganese that are functional in the oxygen-evolving reaction $(4,8-13)$. In support of this view, lightinduced changes in relaxivity under conditions of both steady-state and flash illumination have been reported (8-11).

In our experience, chloroplast thylakoid membranes prepared in buffers that do not contain metal ion chelators (e.g., EDTA) invariably exhibit dramatic changes in the solvent proton relaxation rates upon illumination (6). These effects, which persist for many seconds or minutes after cessation of illumination, can effectively be suppressed by inclusion of $1 \mathrm{mM}$ EDTA in the chloroplast isolation buffer. Furthermore, light-induced relaxation changes reappear when $\mathrm{MnCl}_{2}(50 \mu \mathrm{M}$ final concentration) is added to chloroplasts that have been isolated in the presence of EDTA and subsequently washed free of the chelator (6). From these observations we have concluded that the intrinsic illumination effects arise from paramagnetic ions that can be 
removed by EDTA, a treatment which does not significantly affect water oxidation, electron transport, or photophosphorylation efficiences (5). An understanding of these light-induced effects on relaxivity is essential to an unambiguous interpretation of nuclear magnetic relaxation phenomena in thylakoid membrane suspensions from which nonfunctional $\mathrm{Mn}$ has been removed; the present work further characterizes the changes in relaxivity seen in the presence of nonfunctional $\mathrm{Mn}$, and provides evidence that they arise from oxidation of $\mathrm{Mn}(\mathrm{II})$ by superoxide ion.

\section{MATERIALS AND METHODS}

Chloroplast thylakoid membranes were prepared by a modification of the method of Robinson and Yocum (14) in that $1 \mathrm{mM}$ EDTA was present in the homogenization buffer used in the first step in the isolation procedure. Thylakoid membranes inhibited by treatment with $\mathrm{Hg}$ plus $\mathrm{KCN}$ were prepared according to the procedure of Yocum and Guikema (15). The resuspending buffer contained $0.4 \mathrm{M}$ sucrose, $0.15 \mathrm{M} \mathrm{NaCl}$, and $20 \mathrm{~mm}$ Tricine $(\mathrm{pH} \mathrm{8.0)}$. The NMR instrumentation has been described previously (5-7). Spin-lattice relaxation rates, $R_{1}$, were measured at $25^{\circ} \mathrm{C}$ at $20.7 \mathrm{MHz}$ using the modified triplet sequence (16) and analyzed by a microcomputer (Commodore Model 2001). All of the timed measurement sequences were under microprocessor control. Illumination was conducted by rotating the NMR tube in a beam of intense white light $\left(>10^{6} \mathrm{erg} \cdot \mathrm{cm}^{-2} \cdot \mathrm{s}^{-1}\right)$ filtered through $2.5 \mathrm{~cm}$ of $0.2 \%$ $\mathrm{CuSO}_{4}$ solution in a manner that coated the inner walls of the tube with a thin film of suspension. Deoxygenation, where indicated, utilized a stream of nitrogen gas that had been deoxygenated in a pyrogallate trap and subsequently saturated with water vapor. The stream of wet $\mathrm{N}_{2}$ gas circulated through the NMR tube for $1 \mathrm{~min}$ while the tube was rotated on its side in the dark in the manner described above for illumination. Control NMR experiments showed that this procedure eliminates the contribution to $R_{1}$ from dissolved oxygen.

Catalase and NADPH were obtained from Sigma. Human superoxide dismutase was a generous gift from Dr. J. A. Fee.

\section{RESULTS}

We have previously shown that chloroplast thylakoid membranes isolated in the presence of $1 \mathrm{~mm}$ EDTA are characterized by relatively low relaxation rates $\left(R_{1}=0.7\right.$ to $1.0 \mathrm{~s}^{-1}$ at $\mathrm{chl}$ concentrations of $2-3 \mathrm{mg} / \mathrm{ml}$ ) which do not undergo significant changes upon illumination (6). Light-induced changes in relaxivity are seen only in thylakoid preparations isolated in the absence of EDTA, or in membranes isolated in the presence of EDTA to which $\mathrm{Mn}$ (II) has intentionally been added (6). The latter situation is illustrated by the data shown in Fig. 1A, where addition of $\mathrm{Mn}$ (II) to a final concentration of $50 \mu \mathrm{M}$ is seen to increase threefold the value of $R_{1}$ measured at 20.7 $\mathrm{MHz}$. Subsequent illumination of the sample with strong white light $\left(>10^{6} \mathrm{erg} \cdot \mathrm{cm}^{-2} \cdot \mathrm{s}^{-1}\right)$ produces a decrease in $R_{1}$ measured a few seconds after illumination has ceased. Both the extent of the decrease in $R_{1}$ and the time required for the recovery of the original relaxivity, as illustrated by data in Figs. 1A and $1 \mathrm{~B}$, depend on the length of the illumination period, with longer light exposure (1B) producing a larger initial decrease in $R_{1}$ and a slower dark recovery. The dark recovery kinetics are complex and vary with different preparations of chloroplasts. Under experimental conditions similar to those seen in Fig. 1, but with other chloroplast membrane preparations we have also observed biphasic recovery kinetics (6).

The light-induced changes seen in Fig. 1 arise from exogenously added $\mathrm{Mn}$ (II), rather than from the pool of $\mathrm{Mn}$ associated with oxygen evolution. One explanation for such changes might therefore be an energylinked ion pumping process. It has been shown that cations and anions are translocated into, and out of, illuminated thylakoids in concert with the uptake of protons (17). If exogenously added $\mathrm{Mn}$ (II) were translocated from one side of the thylakoid membrane to the other in the light, substantial changes in $R_{1}$ might be observed if the $\mathrm{Mn}$ (II) binding sites on the two sides of the membrane were different. This possibility was examined by means of experiments similar to those shown in Fig. 1 except in the presence of uncoupling concentrations of gramicidin D. Results very similar to those observed in Fig. 1 were obtained (data not shown), indicating that the light-induced decrease in $R_{1}$ cannot be due to an ion pumping phenomenon coupled to proton translocation.

An alternative explanation which we 


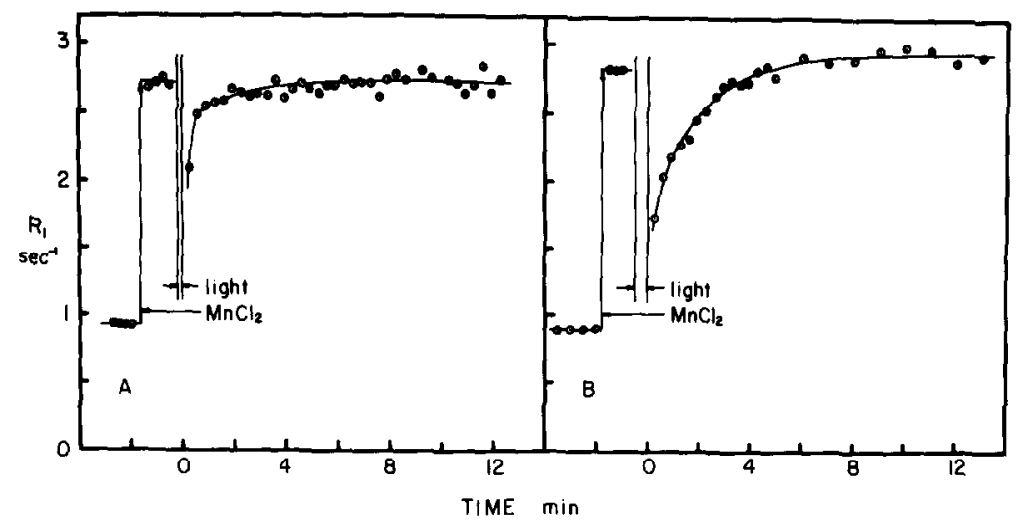

FiG. 1. Changes in the proton magnetic relaxation rate of aerobic chloroplast suspensions (3.0 $\left.\mathrm{mg} \mathrm{chl} \cdot \mathrm{ml}^{-1}\right)$ following addition of $\mathrm{MnCl}_{2}(50 \mu \mathrm{M}$ final concentration) and subsequent illumination in white light $\left(>10^{6} \mathrm{erg} \cdot \mathrm{cm}^{-2} \cdot \mathrm{s}^{-1}\right)$. Measurements were conducted at $25^{\circ} \mathrm{C}$ in the presence of $88 \mu \mathrm{M}$ benzylviologen. (A) 10-s and (B) 30-s illumination.

have proposed previously (6), for the decreases in $R_{1}$ shown in Fig. 1 is that superoxide ion, generated as a product of the reoxidation of low potential reductants associated with PSI might serve as an oxidant of exogenously bound $\mathrm{Mn}$ (II) (1820 ). This possibility is supported by the data shown in Fig. 2; Fig. 2A shows the results of an experiment where superoxide dismutase was included prior to illumination (solid circles). In comparison to a control experiment minus the enzyme (open circles), superoxide dismutase causes a smaller observed decrease in $R_{1}$ as well as a more rapid recovery of the original relaxivity. Note also that the 1-min preillumination period produces even longer recovery times for $R_{1}$ when compared to the data in Fig. 1 . Figure 2B shows that under anaerobic conditions, thylakoid membranes to which DCMU was added (to block oxygen evolution) along with $\mathrm{Mn}$ (II) are devoid of any light-induced changes in $R_{1}$. Since DCMU under aerobic conditions does not interfere with the illumination effect (6), the presence of oxygen is apparently required for the oxidation of $\mathrm{Mn}$ (II) in the light.

Figure 3 presents the results of experiments with thylakoid membranes in which

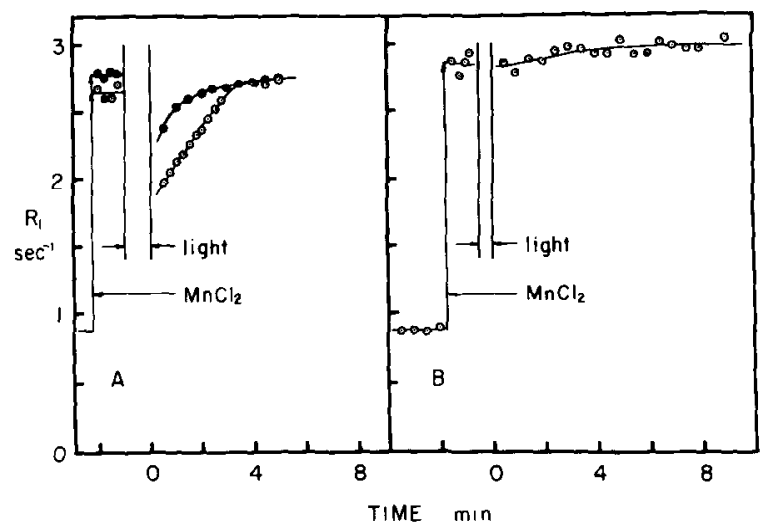

FIG. 2. Changes in the proton magnetic relaxation rate following addition of $\mathrm{MnCl}_{2}(50 \mu \mathrm{M}$ final concentration) and subsequent illumination. (a) 60-s illumination in the presence $(\odot)$ and absence $(\odot)$ of human superoxide dismutase $(0.1 \mathrm{mg} / \mathrm{ml})$; (B) 30 -s illumination under anaerobic conditions in the presence of $50 \mu \mathrm{M}$ DCMU. For other experimental conditions, see the legend of Fig. 1 . 


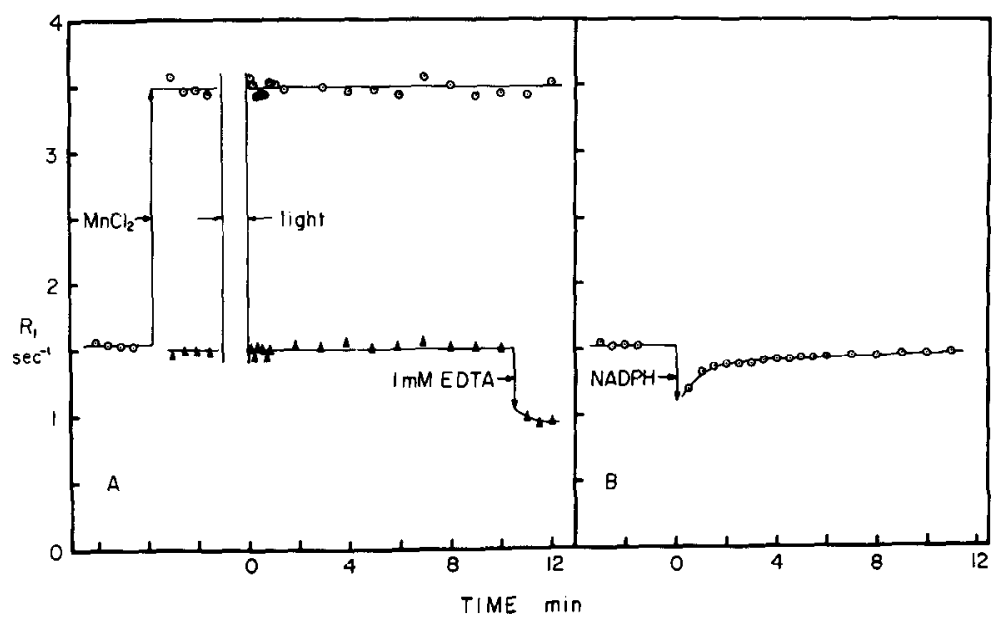

FIG. 3. Changes in the proton relaxation rate of chloroplasts $(2.6 \mathrm{mg} \mathrm{chl} / \mathrm{ml}$, not exposed to EDTA prior to the experiments), that were inhibited by $\mathrm{Hg}^{+2} / \mathrm{CN}^{-}$treatment. (A) $R_{1}$ changes following $60-\mathrm{s}$ illumination in the presence $(\odot)$ and absence $(\triangle)$ of $50 \mu \mathrm{M} \mathrm{MnCl}_{2} ; 50 \mu \mathrm{M}$ methylviologen was present in these experiments. (B) $R_{1}$ changes following additions of $500 \mu \mathrm{M} \mathrm{NADPH}$ in the dark (100 $\mu \mathrm{M}$ benzylviologen added).

plastocyanin function has been inhibited by $\mathrm{KCN} / \mathrm{Hg}$ treatment; this preparation was not exposed to EDTA during isolation. Illumination of these membranes in the absence or presence of added Mn(II) (Fig. $3 \mathrm{~A})$ failed to produce any change in $R_{1}$ (EDTA addition reveals the presence of chelatable paramagnetic ions in the preparation to which exogenous $\mathrm{Mn}$ (II) has not been added; these ions should, if oxidized, produce an effect on $R_{1}$ ). Figure 3B presents results showing that the effect on $R_{1}$ has not been destroyed by $\mathrm{KCN} / \mathrm{Hg}$ treatment. In this experiment generation of a low potential reductant (reduced benzylviologen) was reconstituted by addition of $\mathrm{NADPH}$ and the dye to $\mathrm{KCN} / \mathrm{Hg}$-inhibited thylakoid membranes. As is seen in Fig. $3 \mathrm{~B}$, electron transport from NADPH to oxygen via ferredoxin-NADP reductase and benzylviologen produces an effect on $R_{1}$ similar to that seen in Fig. 1 with illuminated, uninhibited thylakoid membranes.

It is clear from the results in Figs. 1-3 that the light-induced changes in relaxivities of thylakoid membranes containing exogenously added $\mathrm{Mn}$ (II) requires oxygen and an oxidation/reduction reaction associated with PSI and/or the generation of a low potential reductant. The kinetics of the effect on $R_{1}$ are complex and are minimally comprised of oxidation of $\mathrm{Mn}$ (II), presumably by superoxide ion (18-20), as well as subsequent reduction of higher oxidation states of the Mn by reductants, which may include ascorbate (21) retained in our isolated thylakoid membranes. Although we cannot obtain measurements on the initial extent of the decrease in $R_{1}$ (illumination was performed outside the magnet), we can assess the contribution of illumination time to the dark reduction process. From the data in Figs. 1 and 2, it appears that illumination for longer times (up to $1 \mathrm{~min}$ ) tends to decrease the concentration of endogenous compounds which serve to reduce the higher oxidation states of $\mathrm{Mn}$ produced by illumination, thus lengthening the kinetics of manganese rereduction.

A further examination of the change in $R_{1}$ induced by oxidation/reduction reactions is shown in Fig. 4. In 4, a preillumination treatment was first given to deplete endogenous reductants after which an aliquot of NADPH was added to the suspension of thylakoid membranes; $R_{1}$ is seen to decrease to a new value, and then suddenly to rise again. This rapid rise is presumably due to the onset of anaerobiosis in the reaction mixture. Aeration and 


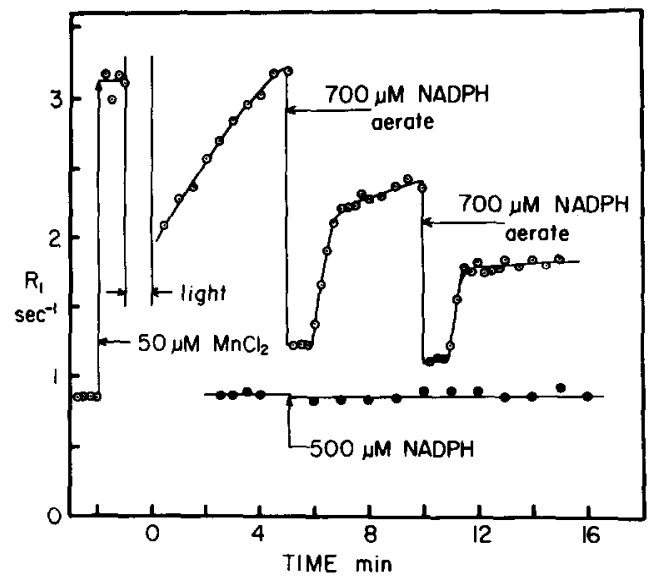

Fig. 4. (๑) $R_{1}$ changes of suspensions containing chloroplasts $\left(3.0 \mathrm{mg} \mathrm{chl} \cdot \mathrm{ml}^{-1}\right)$ and benzylviologen ( 88 $\mu \mathrm{M})$, which were subjected to the following sequence of treatments: addition of $\mathrm{MnCl}_{2}, 60-\mathrm{s}$ illumination, and two additions of NADPH to the final concentrations indicated. (O) $R_{1}$ changes of a chloroplast suspension before and after addition of NADPH to the final concentration indicated. Chloroplasts (equivalent to $3.0 \mathrm{mg} \mathrm{chl} \cdot \mathrm{ml}^{-1}$ ), were isolated in the presence of 1 mM EDTA; measurements were conducted in the absence of an acceptor of added $\mathrm{MnCl}_{2}$.

addition of a second aliquot of NADPH regenerates the decrease in $R_{1}$ followed again by a recovery. Note, however, that as in the preceding experiment, the recovery of $R_{1}$ to the final steady-state value is lower than for the preillumination control. Figure 4 shows that under similar experimental conditions $(500 \mu \mathrm{M}$ NADPH added to chloroplasts isolated in $1 \mathrm{~mm}$ EDTA) but in the absence of added $\mathrm{MnCl}_{2}$, no $R_{1}$ changes are observed.

If NADPH-catalyzed decreases in $R_{1}$ were due to the same process that occurs upon illumination of thylakoid membranes, i.e., oxidation of $\mathrm{Mn}$ (II) by superoxide ion, then the process should be affected by superoxide dismutase. That this is so is shown in Fig. 5A, where inclusion of this enzyme causes a more rapid increase in $R_{1}$ after the initial decrease caused by NADPH addition. Figure $5 \mathrm{~B}$ shows the effect of added catalase on the NADPHcatalyzed effect. Here, the depression in $R_{1}$ persists for slightly longer times than without catalase, an effect which is probably due to regeneration of oxygen from hydrogen peroxide. In some experiments (not shown), the decrease in $R_{1}$ caused by NADPH addition in the presence of catalase persisted for periods up to twice as long as those in the absence of catalase, which supports this hypothesis. Moreover, the fact that catalase does not suppress $R_{1}$ changes indicates that neither $\mathrm{H}_{2} \mathrm{O}_{2}$, nor oxidants generated from $\mathrm{H}_{2} \mathrm{O}_{2}$ as the precursor, such as $\mathrm{OH} \cdot(22)$, are responsible for the observed light-induced effects.

The return of relaxivity seen in Figs. $3-5$ after NADPH addition must be due to a complex series of events including the onset of anaerobiosis, which would decrease

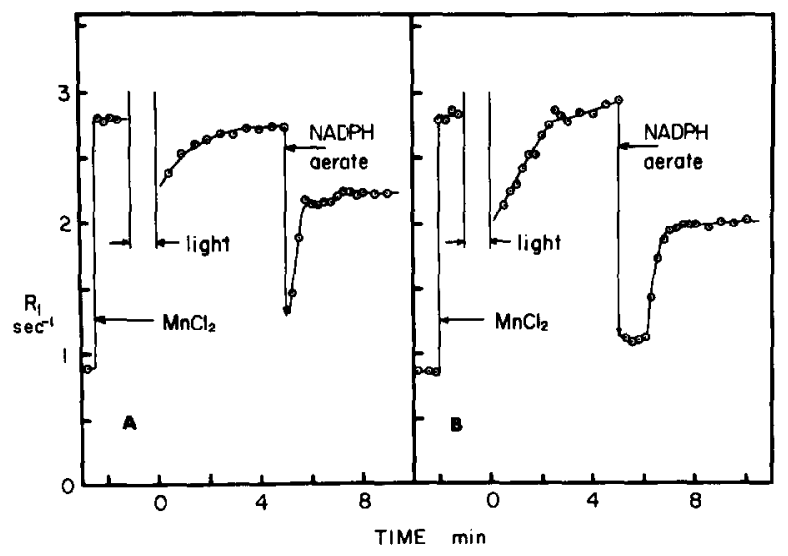

FIG. 5. $R_{1}$ changes of chloroplast following addition of $\mathrm{MnCl}_{2}$, preillumination, and addition of 700 $\mu \mathrm{M}$ NADPH in the presence of (A) human superoxide dismutase $(0.1 \mathrm{mg} / \mathrm{ml}$ ) and (B) catalase $(0.1 \mathrm{mg} / \mathrm{ml})$. For other experimental details see the legend of Fig. 4. 


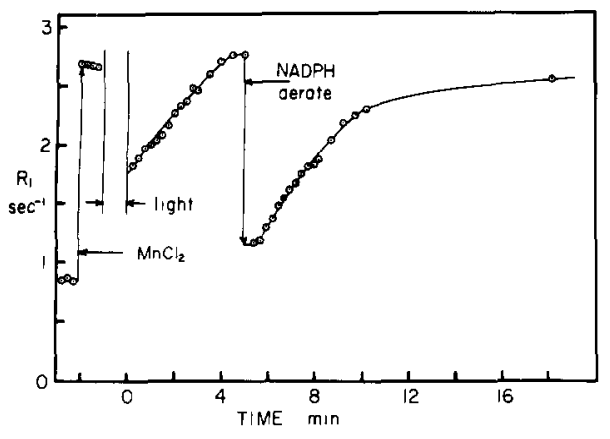

FIG. 6. $R_{1}$ changes of a chloroplast suspension, after addition of $50 \mu \mathrm{M} \mathrm{MnCl}_{2}$, a 60 -s preillumination period, and addition of $300 \mu \mathrm{M}$ NADPH. Chloroplast suspensions $\left(3.0 \mathrm{mg} \mathrm{chl} \cdot \mathrm{ml}^{-1}\right.$ ) contained $88 \mu \mathrm{M}$ benzylviologen.

the concentration of oxidant in the system and would favor rapid rereduction of higher oxidation states of $\mathrm{Mn}$ both by endogenous reductants as well as by reduced benzylviologen, which might accumulate as the system goes anaerobic. When experiments are conducted in the presence of concentrations of NADPH that are insufficient to achieve anaerobiosis, the oxidation poise of the suspension should remain relatively high, and it might be expected that depletion of the redox pool would be much less extensive. Under these conditions, the return of $R_{1}$ to the steady-state value would be less rapid and presumably more similar to the kinetics observed after prolonged aerobic illumination (e.g., Fig. 2). In the presence of significant catalase-like activity, the stoichiometry of NADPH oxidation by ferredoxin-NADP reductase is two NADPH per $\mathrm{O}_{2}$. Since the concentration of $\mathrm{O}_{2}$ in aqueous solution is near $250 \mu \mathrm{M}$ at $25^{\circ} \mathrm{C}$, concentrations of NADPH less than about 500 $\mu \mathrm{M}$ are insufficient to produce anaerobiosis. As shown in Fig. 6, addition of $300 \mu \mathrm{M}$, rather than $700 \mu \mathrm{M}$, NADPH generates a substantial decrease in $R_{1}$, but the recovery of relaxivity is no longer abrupt and in fact quite closely mirrors the recovery after aerobic illumination in the same sample. These observations support the hypothesis that the observed $R_{1}$ changes, especially during the recovery phase, reflect the redox poise of the chloroplast suspension.
Throughout the experiments involving $\mathrm{NADPH}$, it became evident that the reaction scheme (1) outlined in the Discussion does not fully explain the observed $R_{1}$ changes. In addition to the transient depression of $R_{1}$, which we ascribe to $\mathrm{O}_{2}^{--}$ generation and to the subsequent onset of anaerobiosis, there is a secondary $R_{1}$ depression that is essentially irreversible on the time-scale of our experiments. This latter effect is shown clearly in Fig. 7, where NADPH is added to a suspension which was deoxygenated to suppress superoxide formation. The irreversible depression occurs under anaerobiosis in the dark and is dependent upon NADPH concentration. A very similar phenomenon was observed upon addition of NADPH to a solution of mangano-BSA (Fig. 7B), suggesting that a relatively simple physical process is involved, rather than one linked to NADPH oxidoreductase activity. It is well known $(23,24)$ that $\mathrm{Mn}(\mathrm{II})$ chelates tightly to adenine nucleotides through the phosphate esters and through one of the nitrogens, N(7) on the purine moiety. Moreover, we have shown in a previous report (6) that $\mathrm{Mn}^{2+}$ added to chloroplast suspensions is accessible to EDTA, which, like nucleotides, is an anionic chelator ( $\mathrm{p} K \cong 14$ ) and which effectively suppresses $R_{1}$ enhancements. Thus it seems likely that the some-

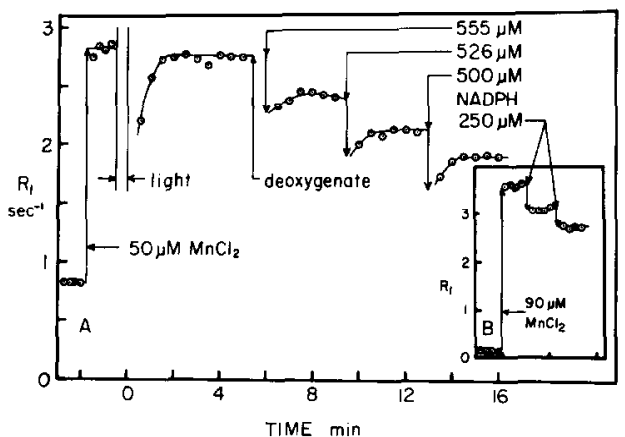

FIG. 7. (A) $R_{1}$ changes in chloroplast suspensions following addition of $\mathrm{MnCl}_{2}, 30$-s illumination, deoxygenation, and addition of three aliquots of NADPH at the final concentrations indicated in the figures. Chloroplasts $\left(3.0 \mathrm{mg} \mathrm{chl} \cdot \mathrm{ml}^{-1}\right)$ contained $88 \mu \mathrm{M}$ benzylviologen. (B) $R_{\mathrm{t}}$ changes of the resuspending buffer plus $30 \mu \mathrm{M}$ bovine serum albumin, following addition of $\mathrm{MnCl}_{2}$ and NADPH at the final concentrations indicated. 
what weaker chelation of $\mathrm{Mn}^{2+}$ by NADPH is responsible for the $R_{1}$ depression seen under anaerobiosis (Fig. 7).

\section{DISCUSSION}

Our previous investigations $(5-7)$ on the origins of the proton spin-lattice relaxation rate $\left(R_{1}\right)$ in thylakoid suspensions have shown that a large, but variable, portion of $R_{1}$ arises from membrane-bound $\mathrm{Mn}(\mathrm{II})$ that is not functional in the water-splitting reaction. Exposure to EDTA effectively suppresses this contribution, which is characterized by a paramagnetic maximum near $20-30 \mathrm{MHz}$ in the field dispersion profile of $R_{1}$. Flash oscillations in $T_{2}{ }^{-1}$ have been reported in experiments using thylakoid membranes that were isolated in the absence of EDTA (8-11); whether such oscillations reflect oxidation state changes of manganese in the oxygen-evolving complex is a question that is not fully resolved at present. We have shown that nonfunctional manganese, which is normally present in such preparations, can produce substantial light-induced variations in $T_{1}^{-1}$, and it is quite possible that these phenomena also underlie the previously observed variations in $T_{2}^{-1}$. It is important to recognize, however, that $T_{2}{ }^{-1}$ is a more complex quantity than $T_{1}{ }^{-1}$ and can in general reflect chemical exchange processes involving paramagnetic sites that do not influence $T_{1}^{-1}$ (1). Nevertheless, the present experiments demonstrate an alternate source of lightinduced relaxivity changes that contribute to both $T_{1}^{-1}$ and $T_{2}{ }^{-1}$ and that do not involve manganese in the oxygen-evolving complex.

In this communication we have extended our earlier observations on the lightinduced $R_{1}$ changes that are observed in thylakoid membranes which contain Mn(II) not associated with the oxygen-evolving apparatus. The requirement for both oxygen (Fig. 2B) and for PSI activity (Fig. 3 ) suggest that $\mathrm{Mn}(\mathrm{II})$ is oxidized by superoxide ion to decrease $R_{1}$ by a mechanism such as that shown below:

$$
\stackrel{\mathrm{O}_{2}}{\longrightarrow} \mathrm{O}_{2}^{-}+2 \mathrm{H}^{+}+\mathrm{Mn}(\mathrm{II}) \longrightarrow \mathrm{Mn}(\mathrm{III})+\mathrm{H}_{2} \mathrm{O}_{2} \text {. }
$$

Reoxidation of the PSI primary acceptor, as well as viologen dyes, by molecular oxygen has been shown to generate superoxide ion $(20,25,26)$. Our data on the effect of superoxide dismutase on both light-induced (Fig. 2A) and NADPHinduced (Fig. $5 \mathrm{~A}$ ) decreases in $R_{1}$ provide further confirmation that superoxide ion is the oxidant of $\mathrm{Mn}$ (II). Our data are in agreement with those presented by Kono et al. (27), who showed by other techniques that superoxide ion produced by photosynthetic electron transport generated $\mathrm{Mn}(\mathrm{III})$, which was originally added to the thylakoid suspensions as Mn(II).

The phenomena which lead to the reduction of higher oxidation states of $\mathrm{Mn}$ generated by superoxide ion are complex and have not fully been characterized in the present study. Thylakoids are known to contain endogenous reductants including ascorbate (21) and other unknown compounds such as those described by Cramer and Butler (28). It is clear from the data that illumination of thylakoids plus Mn(II) in strong white light (Figs. 1, 2, 6) serves to decrease the influence of these reductants in restoring $R_{1}$ to its original value. Furthermore, in the presence of NADPH and benzylviologen, a rapid recovery of $R_{1}$ is seen which may be due to both the onset of anaerobiosis and the presence of endogenous and added (benzylviologen) reductants. The precise identity of the endogenous reductants is beyond the scope of this study.

The studies described here and elsewhere (5-7) indicate that light-induced $R_{1}$ changes observed in chloroplast suspensions reflect oxidation state changes of extraneous paramagnetic ions rather than redox changes of functional components of the electron transport chain. We have shown elsewhere (7) that systematic illumination protocols and chemical treatments that affect the redox poise of the electron 
transport chain do not significantly affect $R_{1}$. Furthermore, the absence of illumination effects on $R_{1}$ under conditions in which PSII is fully functional, but in which reaction (1) above is inhibited, indicates that manganese associated with physiologically relevant $\mathrm{S}$-states is not monitored in our experiments. The mechanism (1) described above must also have contributed to $T_{2}^{-1}$ variations observed during flash sequences (8-11), and further studies of flash oscillations in $T_{2}{ }^{-1}$ under conditions in which superoxide production is inhibited (e.g., anaerobiosis) and in which chelatable manganese has been removed would appear to be warranted. On the other hand, exposure of thylakoid membranes to certain inactivators of water oxidation $\left(\mathrm{NH}_{2} \mathrm{OH}\right.$ $(6,7)$ or Tris (H. H. Robinson, R. R. Sharp, and C. F. Yocum, unpublished observations) in the absence of EDTA) does produce substantial relaxation enhancements, and we have shown (7) that these enhancements arise from manganese associated with the oxygen-evolving reaction. Extensive investigations of the nature of these latter reactions are now in progress.

\section{REFERENCES}

1. DWEK, R. A. (1975) NMR in Biochemistry, Oxford Univ. Press, Oxford.

2. Mildvan, A. S., AND CoHn, M. (1970) Advan. Enzymol. Relat. Areas. Mol. Biol. 33, 1-70.

3. KoENIG, S. H., BROWN, R. D., AND STUDEBAKER, J. (1971) Cold Spring Harbor Symp. Quant. Biol. 36, 551-559.

4. WYDRzYNSKI, T. J., MARKS, S. B., SCHMidT, P. G., GovindJEE, AND GUTOWSKY, H. S. (1978) Biochemistry 17, 2155-2162.

5. SHARP, R. R., AND YoCUM, C. F. (1980) Biochim. Biophys. Acta 592, 185-195.

6. Robinson, H. H., ShaRP, R. R., and Yocum, C. F. (1980) Biochem. Biophys. Res. Commun. 93, 755-761.

7. Robinson, H. H., Sharp, R. R., AND Yocum, C. F. (1980) Biochim. Biophys. Acta 593, 414426.

8. WYDRZYNSKI, T., Zumbulyadis, N., SchmidT, P. G., GuTOWSKY, H. S., AND GovindJEE
(1976) Proc. Nat. Acad. Sci. USA 73, 1196-1198.

9. WYdRZYNSKI, T., GovindJeE, ZumbUlyadis, N., SchmidT, P. G., AND Gutowsky, H. S. (1976) Amer. Chem. Soc. Symp. Ser. 34, 471-482.

10. Govindjee, Wydrzynski, T., AND Marks, S. B. (1977) in Photosynthetic $\mathrm{O}_{2}$ Evolution (Metzner, H., ed.), pp. 321-324, Academic Press, New York.

11. GovindJEE, WYDRZYNSKI, T., AND MakKS, S. B. (1977) in Bioenergetics of Membranes (Packer, L., Papageorgiou, G., and Trebst, A., eds.), pp. 305-316, Elsevier-North Holland, Amsterdam.

12. Marks, S. B., Wydrzynski, T., GovindJee, SCHMIDT, P. G., AND GUTOWSKY, H. S. (1978) in Biomolecular Structure and Function (Ayris, P. F. ed.), pp. 95-100, Academic Press, New York.

13. Gribova, Z. P., Zakharova, N. I., and MURza, L. I. (1978) Sov. Mol. Biol. 12, 157-164.

14. Robinson, H. H., AND Yocum, C. F. (1980) Biochim. Biophys. Acta 590, 97-106.

15. Yocum, C. F., AND Guikema, J. A. (1977) Plant Physiol. 59, 33-37.

16. Sharp, R. R. (1972) J. Chem. Phys. 12, 5321-5330.

17. Hind, G., Nakatani, H. Y., and Izawa, S. (1974) Proc. Nat. Acad. Sci. USA 71, 1484-1488.

18. EPEL, B. L., AND NeumanN, J. (1973) Biochim. Biophys. Acta 325, 520-529.

19. Lumsden, J., AND HaLL, D. O. (1975) Biochem. Biophys. Res. Commun. 64, 595-602.

20. Allen, J. F. (1977) in Superoxide and Superoxide Dismutases (Michelson, A. M., McCord, J. M., and Fridovich, I., eds.), 417-436, Academic Press, London.

21. WALKER, D. A. (1971) in Methods in Enzymology (San Pietro, A., ed.), Vol. 23, pp. 211-220, Academic Press, New York.

22. Beauchamp, C., and Fridovich, I. (1970) $J$. Biol. Chem. 245, 4641-4646.

23. Kotowicz, G., and HayamizU, K. (1973) Biochemistry 12, 517-520.

24. Lam, Y., Kuntz, G. P. P., AND Kotowicz, G. (1973) J. Amer. Chem. Soc. 96, 1834-1839.

25. Elstner, E. F., Stoffer, C., ANd Heupel, A. (1975) $Z$. Naturforsch. 30, 53-56.

26. Elstner, E. F., AND Heupel, A. (1974) $Z$. Naturforsch. 29, 559-563; 564-571.

27. Kono, Y., TAKahashi, M., AND ASADA, K. (1976) Arch. Biochem. Biophys. 174, 454-462.

28. Cramer, W. A., AND Butler, W. (1969) Biochim. Biophys. Acta 172, 503-510. 